\title{
The Emergence of Aberration Correctors for Electron Lenses.
}

\author{
John Silcox
}

\section{School of Applied and Engineering Physics, Cornell University, Ithaca NY 14853}

In 1936, Otto Scherzer published his famous theoretical paper ${ }^{1}$ on the correction of aberrations in round magnetic electron lenses. For fundamental reasons, the aberration coefficients are positive and to introduce negative corrections, the electron optical elements have to produce either charges or poles on the optic axis, or contain a mirror or be a multipole system. In 1997, three papers appeared with actual demonstrations of the correction of aberrations in imaging lenses, ${ }^{2}$, probe forming lenses ${ }^{3}$ and electron mirrors. ${ }^{4}$ It took almost two thirds of a century for this seemingly straightforward development to occur and for the promise of wavelength limited electron imaging to emerge as a practical proposition. In this paper I will put this achievement into perspective, i.e., try to explain why this was so difficult, give some indication of the implications for our field and provide a sense of the contributions of two scientists, Albert Crewe and Harald Rose, whose work has immeasurably influenced the work we do now and, in my view, has even more influenced the future of this field.

Before aberration correction can actually have an impact, the electron optical systems should be operating at or near the theoretical limits. The first instrument that I encountered (in 1961), the Siemens Elmiskop 1A, had a spatial resolution of $15 \AA$. Fortunately, this was more than adequate for the identification and study of dislocations and other important microstructural features in materials. Environmental and manufacturing capabilities almost certainly determined the final performance levels of this instrument. Given the $100 \mathrm{keV}$ electron wavelength of $0.037 \AA$, the $15 \AA$ value was so far away from the performance limit that serious improvements in design, manufacturing and environment of the basic instrument had to happen before aberration correction could be considered essential to further progress in electron microscopy. In other words there was plenty to do with the existing instrument.

These considerations did not prevent efforts at understanding how to do this and in looking for neat ways to circumvent the aberration limitations. For example, Denis Gabor invented optical holography (and won the 1971 Nobel Prize for physics) as a means of correcting electron micrographs for aberration distortions. Only very recently have serious efforts been undertaken to seriously exploit electron holography as a method of improving information at the atomic scale (See, for example the symposium at this meeting in honor of Hannes Lichte and Akira Tonomura). The prospect of atomic scale imaging resolution, albeit a distant goal, still served to stimulate efforts to circumvent Electron optics was undertaken as a study in its own right and substantial commitments of time, funds and ingenuity were expended in making progress towards this eventual goal. Thus a serious background to the subject was developed even though working electron microscopes did not operate at the level that was limited by the electron optical performance of the lenses.

I date the modern era from the arrival of Albert Crewe on the electron microscopy scene in the late to early seventies. Professor Crewe made his reputation in accelerator physics and used insights from his experience in that field in his venture into electron optics. On becoming Director of Argonne National Laboratory, the story goes that on wandering through the laboratory he was struck by how many of the staff used electron microscopy and wanted to help. In the intervening period 
both at Argonne and later at the University of Chicago, he and his group introduced the field emission gun that is now common, added a high quality (i.e., small spherical aberration coefficient) lens to form a high resolution scanning transmission electron microscope (STEM), used an Annular Dark Field detector for "Z-contrast" imaging and added an electron spectrometer for Electron Energy Loss Spectrometry. In this period atoms were imaged for the first time and in short he and his team changed the field of electron microscopy as we know it today. For a period, that team included Elmer Zeitler and Harald Rose as well as a number of bright graduate students who have gone on to make their own independent marks.

Once the new instrument had been established, serious work began on the very difficult problem of correcting aberrations to reduce the probe even more. Work was under way in several laboratories around the world in the UK, Germany and US throughout this period. Space forbids an exhaustive survey in this abstract so only the work of Jiye and Crewe analyzing the electron optics of multipole systems and sextupole/roundlenses/sextupole ${ }^{5}$ systems will be noted here.

In the meantime, Harald Rose went back to Germany and took up the chair in Darmstadt following in the long history and tradition established by Otto Scherzer. There he has trained a number of students in electron optics (such as Max Haider ${ }^{2}$ ) and has been particularly concerned with the problems of practical construction of the many devices invented by himself and his students. His 1990 paper $^{6}$ laid out in detail the design of a spherically corrected semi-aplanatic TEM that was the harbinger of the instrument later reported.

In the final analysis, this has been a long path to realization and almost certainly, had to await the arrival of the computational capability as applied in simulation of the optical paths through the lenses, control of the machining capability and control of the lens excitations and overall operations.

Has it been worth it? I am an unabashed advocate of the new era and look forward eagerly to these instruments.

\section{References}

[1] O. Scherzer, Z.Physik 101, 593-603 (1936).

[2] M. Haider, S. Uhlemann, E. Schwan, H.Rose, B. Kabius, K. Urban, Nature, 392, 768-769 (1998).

[3] O.L. Krivanek, N. Dellby, A.J.H. Spence, R.A. Camps and L.M. Brown, EMAG '97 I.o.P.

Conference series (ed. J.M.Rodenburg) 153, 35-40 (1997).

[4] G.F. Rempfer, D.M. Desloge, W.P. Skoczylas, and O.H. Griffith, Microscopy and Microanalysis 3, 14-27 (1997).

[5] X. Jiye and A.V. Crewe, Optik 70 37-42 (1985)

[6] H. Rose, Optik 85 19-24 (1990). 